\title{
A170 ANTI-CD20 ANTIBODY-MEDIATED APOPTOSIS OF B CELLS IS A LIPID RAFT-DEPENDENT PROCESS
}

Jacques-Oliver Pers, ${ }^{1}$ Mariam Hammadi, ${ }^{1}$ Florence Dalbies, ${ }^{2}$ Adrian Tempescul, ${ }^{2}$ Christian Berthou, 1,2 Anne Bordron,1,2 Pierre Youinou' ${ }^{1} E A 2216$ 'Immunology \& Pathology', Brest University Medical School Hospital, Brest, France; '2Department of Haematology and Oncology, Brest University Medical School Hospital, Brest, France

\subsection{6/ard.2010.149013.13}

Background $\mathrm{B}$ cell depletion has become a classics in approved conditions and off-label indications. Anti-CD20 have been classified into type-I, such as rituximab (RTX), and type-II monoclonal antibodies (mAbs), such as tositumomab (B1). Since the effects of RTX are variable in autoimmune diseases, the authors have tested those of B1 on the easily accessible B cells of chronic lymphocytic leukaemia (CLL) and marginal zone lymphoma (MZL). This $\mathrm{mAb}$ requires translocation of its target into lipid rafts (LR) containing cholesterol, sphingomyelin (SM), ganglioside M1 (GM1) and proteins associated such as $\mathrm{Cbp} / \mathrm{P}$-glycoprotein (PAG) implicated in signalling.

Material and methods B lymphocytes were obtained from 36 untreated CLL patients and 8 MZL used as disease controls. B cells were cultured in 24 -well plates at $37^{\circ} \mathrm{C}$ with or without B1. Apoptosis was investigated using annexin V/ propidium iodide. GM1 and SM, were quantified by flow cytometry and Western blotting as well as Cbp/PAG and PGP. Modulation activity of tositumomab by rifampicin, an activator of Pgp was visualised by annexin V/propidium iodide. Characterisation of LR-associated proteins was performed by confocal microscopy.

Results B1-binding induced B cell apoptosis in 50\% of CLLs, and in all MZL tested. A higher CD20 expression was not correlated with sensitivity to B1-induced apoptosis. In fact, apoptosis was associated with a higher GM1 and extramembrane expression of SM leading to the recruitment of Cbp/PAG. The authors observed by confocal microscopy that, in this case, CD20, SM and Cbp/PAG were colocalized.

In contrast, those CLL B cells resisting B1-mediated apoptosis, sequestrated SM in the cytoplasm, due to a decrease of Pgp activity. Pgp are indeed implicated in SM exportation to the outer leaflet of the membrane and can be activated by rifampicin. Restored Pgp activity with rifampicin in anti-CD20 resistant $B$ cell leads to an increased location of SM at the outer side of the cell membrane, the recruitment of Cbp/PAG and the induction of apoptosis.

Conclusions This data supports the view of a fine regulation of lipids implicated in survival or apoptotic signals in B cells. Acting on the lipid distribution at the cell membrane will permit to improve anti-CD20 mAb efficacy. 